\title{
Increasing the Effectiveness of Active Learning: Introducing Artificial Data Generation in Active Learning for Land Use/Land Cover Classification
}

\author{
Joao Fonseca *(D), Georgios Douzas (D) and Fernando Bacao (D) \\ Campus de Campolide, NOVA Information Management School (NOVA IMS), Universidade Nova de Lisboa, \\ 1070-312 Lisboa, Portugal; gdouzas@novaims.unl.pt (G.D.); bacao@novaims.unl.pt (F.B.) \\ * Correspondence: jpfonseca@novaims.unl.pt
}

check for

updates

Citation: Fonseca, J.; Douzas, G.; Bacao, F. Increasing the Effectiveness of Active Learning: Introducing Artificial Data Generation in Active Learning for Land Use/Land Cover Classification . Remote Sens. 2021, 13, 2619. https://doi.org/10.3390/ rs13132619

Academic Editors: Danfeng Hong and Dino Ienco

Received: 26 April 2021

Accepted: 30 June 2021

Published: 4 July 2021

Publisher's Note: MDPI stays neutral with regard to jurisdictional claims in published maps and institutional affiliations.

Copyright: (c) 2021 by the authors. Licensee MDPI, Basel, Switzerland. This article is an open access article distributed under the terms and conditions of the Creative Commons Attribution (CC BY) license (https:/ / creativecommons.org/licenses/by/ $4.0 /)$.

\begin{abstract}
In remote sensing, Active Learning (AL) has become an important technique to collect informative ground truth data "on-demand" for supervised classification tasks. Despite its effectiveness, it is still significantly reliant on user interaction, which makes it both expensive and time consuming to implement. Most of the current literature focuses on the optimization of AL by modifying the selection criteria and the classifiers used. Although improvements in these areas will result in more effective data collection, the use of artificial data sources to reduce human-computer interaction remains unexplored. In this paper, we introduce a new component to the typical AL framework, the data generator, a source of artificial data to reduce the amount of user-labeled data required in AL. The implementation of the proposed AL framework is done using Geometric SMOTE as the data generator. We compare the new AL framework to the original one using similar acquisition functions and classifiers over three AL-specific performance metrics in seven benchmark datasets. We show that this modification of the AL framework significantly reduces cost and time requirements for a successful AL implementation in all of the datasets used in the experiment.
\end{abstract}

Keywords: active learning; artificial data generation; land use/land cover classification; oversampling; SMOTE

\section{Introduction}

The technological development of air and spaceborne sensors and the increasing number of remote sensing missions have allowed the continuous collection of large amounts of high quality remotely sensed data. These data are often composed of multi- and hyperspectral satellite imagery, essential for numerous applications, such as Land Use/Land Cover (LULC) change detection, ecosystem management [1], agricultural management [2], water resource management [3], forest management, and urban monitoring [4]. Despite LULC maps being essential for most of these applications, their production is still a challenging task $[5,6]$. They can be updated using one of the following strategies:

1. Photo-interpretation. This approach consists of evaluating a patch's LULC class by a human operator based on orthophoto and satellite image interpretation [7]. This method guarantees a decent level of accuracy, as it is dependent on the interpreter's expertise and human error. Typically, it is an expensive, time-consuming task that requires the expertise of a photo-interpreter. This task is also frequently applied to obtain ground-truth labels for training and/or validating Machine Learning (ML) algorithms for related tasks [8,9].

2. Automated mapping. This approach is based on the usage of a ML method or a combination of methods in order to obtain an updated LULC map. The development of a reliable automated method is still a challenge among the ML and remote sensing community, since the effectiveness of existing methods varies across applications and geographical areas [5]. Typically, this method requires the existence of ground-truth 
data, which are frequently outdated or nonexistent for the required time frame [1]. On the other hand, employing a ML method provides readily available and relatively inexpensive LULC maps. The increasing quality of state-of-the-art classification methods has motivated the application and adaptation of these methods in this domain [10].

3. Hybrid approaches. These approaches employ photo-interpreted data to augment the training dataset and improve the quality of automated mapping [11]. They attempt to accelerate the photo-interpretation process by selecting a smaller sample of the study area to be interpreted. The goal is to minimize the inaccuracies found in the LULC map by supplying high-quality ground-truth data to the automated method. The final (photo-interpreted) dataset consists of only the most informative samples, i.e., patches that are typically difficult to classify for a traditional automated mapping method [12].

The latter method is best known as AL. It is especially useful whenever there is a shortage or even absence of ground-truth data and/or the mapping region does not contain updated LULC maps [13]. In a context of limited sample-collection budget, the collection of the most informative samples capable of optimally increasing the classification accuracy of a LULC map is of particular interest [13]. AL attempts to minimize the human-computer interaction involved in photo-interpretation by selecting the data points to include in the annotation process. These data points are selected based on an uncertainty measure and represent the points close to the decision borders. Afterwards, they are passed on for photo-interpretation and added to the training dataset, while the points with the lowest uncertainty values are ignored for photo-interpretation and classification. This process is repeated until a convergence criterion is reached [14].

The relevant work developed within AL is described in detail in Section 2. This paper attempts to address some of the challenges found in AL, mainly inherited from automated and photo-interpreted mapping: mapping inaccuracies and time consuming human-computer interactions. These challenges have different sources:

1. Human error. The involvement of photo-interpreters in the data labeling step carries an additional risk to the creation of LULC patches. The minimum mapping unit being considered and the quality of the orthophotos and satellite images being used are some of the factors that may lead to the overlooking of small-area LULC patches and label-noisy training data [15].

2. High-dimensional datasets. Although all the bands (i.e., features) present in multiand hyperspectral images contain useful information for automated classification, they also introduce an increased level of complexity and redundancy in the classification step [16]. These datasets are often prone to the Hughes phenomenon, also known as the curse of dimensionality.

3. Class separability. Producing an LULC map considering classes with similar spectral signatures makes them difficult to separate [17]. A lower pixel resolution of the satellite images may also imply mixed-class pixels, which may lead to both lower class separability and higher risk of human error.

4. Existence of rare land cover classes. The varying morphologies of different geographical regions naturally implies an uneven distribution of land cover classes [18]. This is particularly relevant in the context of AL since the data selection method is based on a given uncertainty measure over data points whose class label is unknown. Consequently, AL's iterative process of data selection may disregard wrongly classified land cover areas belonging to a minority class.

Research developed in the field of AL typically focuses on the reduction of human error by minimizing the human interaction with the process through the development of more efficient classifiers and selection criteria within the generally accepted AL framework. Concurrently, the problem of rare land cover classes is rarely addressed. This is a frequent problem in the ML community, known as the Imbalanced Learning problem. This problem 
exists whenever there is an uneven between-class distribution in the dataset [19]. Specifically, most classifiers are optimized and evaluated using accuracy-like metrics, which are designed to work primarily with balanced datasets. Consequently, these metrics tend to introduce a bias towards the majority class by attributing an importance to each class proportional to its relative frequency [10]. For example, such a classifier could achieve an overall accuracy of $99 \%$ on a binary dataset where the minority class represents $1 \%$ of the overall dataset and thus still be useless. Several methods have been developed to deal with this problem. They can be categorized into three different types of approaches [20,21]. Cost-sensitive solutions perform changes to the cost matrix in the learning phase. Algorithmic level solutions modify specific classifiers to reinforce learning on minority classes. Resampling solutions modify the training data by removing majority samples and/or generating artificial minority samples. The latter is independent from the context and can be used alongside any classifier. Since we are interested in the introduction of artificial data generation in AL, we analyze the state of the art on resampling techniques (specifically oversampling) in Section 3.

In this paper, we propose a novel AL framework to address two limitations commonly found in the literature: minimize human-computer interaction and reduce the class imbalance bias. This is done with the introduction of an additional component in the iterative AL procedure (the generator) that is used to generate artificial data to both balance and augment the training dataset. The introduction of this component is expected to reduce the number of iterations required until the classifier reaches a satisfactory performance.

This paper is organized as follows. Section 1 explains the problem and its context. Sections 2 and 3 describe the state of the art in AL and oversampling techniques. Section 4 explains the proposed method. Section 5 covers the datasets, evaluation metrics, ML classifiers, and experimental procedure. Section 6 presents the experiment's results and discussion. Section 7 presents the conclusions drawn from our findings.

\section{Active Learning Approaches}

As the amount of unlabeled data increases, the interest and practical usefulness of AL follows that trend [22]. AL is used as the general definition of frameworks aiming to train a learning system in multiple steps, where a new dataset is chosen and added to the training dataset each time [11]. Typically, an AL framework is composed of the following elements [11,13,23]:

1. Unlabeled dataset. It consists of the original data source (or a sample thereof). It is used in combination with the chooser and the selection criterion to expand the training dataset in regions where the classification uncertainty is higher. Therefore, the unlabeled dataset is used for both producing the initial training dataset by selecting a set of instances for the supervisor to annotate (discussed in point 3) and calculating the uncertainty map to augment the training dataset.

2. Supervisor. It is a human annotator (or team of human annotators) to whom the uncertainty map is presented. The supervisor is responsible for annotating unlabeled instances to be added to the augmented dataset. In remote sensing, the supervisor is typically a photo-interpreter, as is the case in [24]. Some research also refers to the supervisor as the oracle [11,25-27].

3. Initial training dataset. It is a small, labeled sample of the original data source used to initiate the first AL iteration. The size of the initial training sample normally varies between no instances at all and $10 \%$ of the unlabeled dataset [28].

4. Current and expanded training dataset. It is the concatenation of the initial training dataset and the datasets labeled by the supervisor in past iterations (discussed in Point 2).

5. Chooser (classifier). It produces the class probabilities for each unlabeled instance.

6. Selection criterion. It quantifies the chooser's uncertainty level for each instance belonging to the unlabeled dataset. It is typically based on the class probabilities assigned by the chooser. In some situations, the chooser and the selection criterion 
are grouped together under the concept acquisition function [11] or query function [13]. Some of the literature refers to the selection criterion by using the concept sampling scheme [12].

Figure 1 schematizes the steps involved in a complete AL iteration. For a better context within the remote sensing domain, the prediction output can be identified as the LULC map. This framework starts by collecting unlabeled data from the original data source. They are used to generate a random initial training sample and are labeled by the supervisor. In practical applications, the supervisor is frequently a group of photo-interpreters [22]. The chooser is trained on the resulting dataset and is used to predict the class probabilities on the unlabeled dataset. The class probabilities are fed into a selection criterion to estimate the prediction's uncertainty, out of which the instances with the highest uncertainty will be selected. This calculation is motivated by the absence of labels in the uncertainty dataset. Therefore, it is impossible to estimate the prediction's accuracy in the unlabeled dataset in a real case scenario. The iteration is completed when the selected points are tagged by the supervisor and added to the training dataset (i.e., the augmented dataset).

Initialization

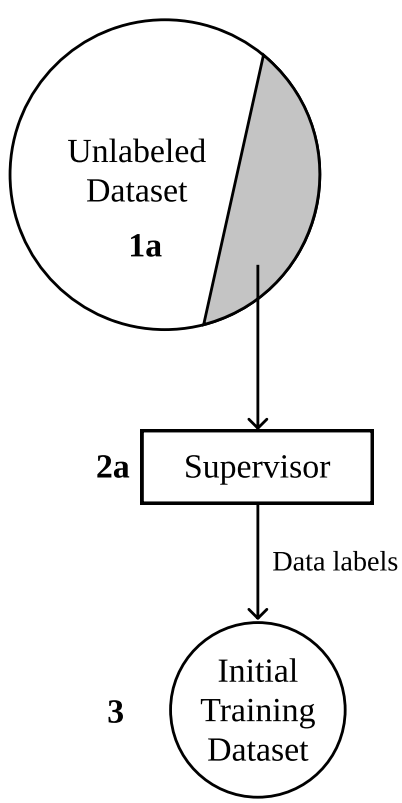

Iteration

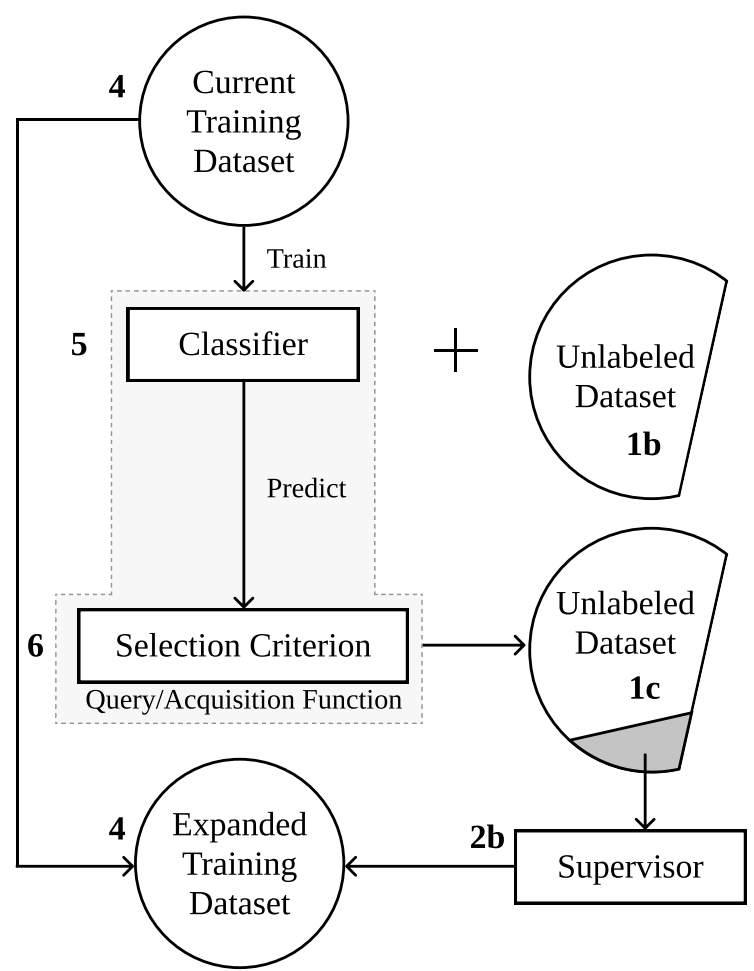

Figure 1. Diagram depicting the typical AL framework.

A common challenge found in AL tasks is ensuring the consistency of AL over different initializations [22]. There are two factors involved in this phenomenon. On the one hand, the implementation of the same method over different initializations may result in significantly different initial training samples, leading to varying accuracy curves. On the other hand, the lack of a robust selection criterion and/or classifier may also result in inconsistencies across AL experiments with different initializations. This phenomenon was observed and documented in a LULC classification context in [29].

The classification method plays a central role in the efficacy of AL. The classifier used should be able to generalize with a relatively small training dataset. Specifically, deep learning models are used in image classification due to their capability of producing high quality predictions. However, to make such models generalizable, the training set must be large enough, making its suitability for AL applications an open challenge [30-32]. Some 
studies in the remote sensing domain were developed to address this gap. In [30,32], the authors proposed a deep learning-based AL approach by training the same convolutional neural network incrementally across iterations and smoothening the decision boundaries of the model using the Markov random field model and a best-versus-second best labeling approach. This allows the introduction of additional data variability in the final training dataset. Wu et al. [31] combined transfer learning, active classification and segmentation techniques for vehicle detection. By combining different techniques, they were able to produce a classification mechanism that performed well when the amount of training data is limited. However, the exploration of advanced deep learning classifiers in AL is still limited. In [33], the authors showed that deep learning classifiers performs well on LULC classification, but are still not generalizable for different geographical regions or periods. Specifically, AL methods are still incapable of providing generalizable deep learning classifiers, which benefit from multiple advantages. The development of convolutional neural networks with both two- and three-dimensional convolutions was explored by Roy et al. [34] who reported superior classification performance on benchmark datasets. However, many training data were used to produce the final classification map.

Selecting an efficient selection criterion is particularly important to find the instances closest to the decision border (i.e., instances difficult to classify) [35]. Therefore, many AL related studies focus on the design of the query/acquisition function [13].

\subsection{Non-Informed Selection Criteria}

Only one non-informed (i.e., random) selection criterion was found in the literature. Random sampling selects unlabeled instances without considering any external information produced by the chooser. Since the method for selecting the unlabeled instances is random, this method disregards the usage of a chooser and is comparatively worse than any other selection criterion. However, random sampling is still a powerful baseline method [27].

\subsection{Ensemble-Based Selection Criteria}

Ensemble disagreement is based on the class predictions of a set of classifiers. The disagreement between all the predictions for a given instance is a common measure for uncertainty, although computationally inefficient [11,14]. It is calculated using the set of classifications over a single instance, given by the number of votes assigned to the most frequent class [35]. This method was implemented successfully for complex applications such as deep active learning [11].

Multiview [36] consists on the training of multiple independent classifiers using different views, which correspond to the selection of subsets of features or instances in the dataset. Therefore, it can be seen as a bootstrap aggregation (bagging) ensemble disagreement method. It is represented by the maximum disagreement score out of a set of disagreements calculated for each view [35]. A lower value for this metric means a higher classification uncertainty. Multiview-based maximum disagreement has been successfully applied to hyperspectral image classification [37,38].

An adapted disagreement criterion for an ensemble of $k$-nearest neighbors is proposed in [14]. This method employs a $k$-nearest neighbors classifier and computes an instance's classification uncertainty based on the neighbors' class frequency using the maximum disagreement metric over varying values for $k$. As a result, this method is comparable to computing the dominant class' score over a weighted $k$-nearest neighbors classifier. This method was also used on a multimetric active learning framework by Zhang et al. [39].

Another relevant ensemble-based selection criterion is the binary random forest-based query model [13]. This method employs a one-versus-one ensemble method to demonstrate an efficient data selection method using the estimated probability of each binary random forest and determining the classification uncertainty based on the probabilities closest to 0.5 (i.e., the least separable pair of classes is used to determine the uncertainty value). 
However, this study fails to compare the proposed method with other benchmark methods, such as random sampling.

\subsection{Entropy-Based Criteria}

Several contributions have focused on entropy-based querying. The application of entropy is common among active deep learning applications [26], where the training of an ensemble of classifiers is often too expensive.

Entropy Query-by-Bagging (EQB), also defined as maximum entropy [12], is an ensemble approach of the entropy selection criterion, originally proposed in [40]. This strategy uses the set of predictions produced by the ensemble classifier to calculate the many entropy measurements. The estimated uncertainty measure for one instance is given by the maximum entropy within that set. EQB has been observed to be an efficient selection criterion. Specifically, Shrivastava and Pradhan [35] applied EQB on hyperspectral remote sensing imagery using Support Vector Machines (SVM) and Extreme Learning Machines (ELM) as choosers, achieving optimal results when combining EQB with ELM. Another study successfully implemented this method on an active deep learning application [12]. Another study improved over this method with a normalized EQB selection criterion [41].

\subsection{Other Relevant Criteria}

Margin Sampling is a SVM-specific criterion, based on the distance of a given point to the SVM's decision boundary [35]. This method is less popular than the remaining methods because it is limited to one type of chooser (SVMs). One extension of this method is the multi-class level uncertainty [35], calculated by subtracting the instance's distance to the decision boundaries of the two most probable classes [42].

The Mutual Information-based (MI) criterion selects the new training instances by maximizing the mutual information between the classifier and class labels in order to select instances from regions that are difficult to classify. Although this method is commonly used, it is frequently outperformed by the breaking ties selection criterion [43,44].

The breaking ties (BT) selection criterion was originally introduced by Luo et al. [45]. It consists of the subtraction between the probabilities of the two most likely classes. Another related method is Modified Breaking Ties scheme (MBT), which aims at finding the instances containing the largest probabilities for the dominant class [44,46]

Another type of selection criteria identified is the loss prediction method [25]. This method replaces the selection criterion with a predictor whose goal is to estimate the chooser's loss for a given prediction. This allows the new classifier to estimate the prediction loss on unlabeled instances and select the ones with the highest predicted loss.

Some of the literature fails to specify the strategy employed, although inferring it is generally intuitive. For example, Ertekin et al. [47] successfully used AL to address the imbalanced learning problem. They employed an ensemble of SVMs as the chooser, as well as an ensemble-based selection criterion. All of the research found related to this topic focuses on the improvement of AL through modifications on the selection criterion and classifiers used. None of these publications propose significant variations to the original AL framework.

\section{Artificial Data Generation Approaches}

The generation of artificial data is a common approach to address imbalanced learning tasks [21], as well as improve the effectiveness of supervised learning tasks [48]. In recent years, some sophisticated data generation approaches have been developed. However, the scope of this work is to propose the integration of a generator within the AL framework. To do this, we focus on heuristic data generation approaches, specifically, oversamplers.

Heuristic data resampling methods employ local and/or global information to generate new, relevant, non-duplicate instances. These methods are most commonly used to populate minority classes and balance the between-class distribution of a dataset. The Synthetic Minority Oversampling Technique (SMOTE) [49] is a popular heuristic oversampling 
algorithm, proposed in 2002. The simplicity and effectiveness of this method contributes to its prevailing popularity. It generates a new instance through a linear interpolation of a randomly selected minority-class instance and one of its randomly selected $k$-nearest neighbors. The implementation of SMOTE for LULC classification tasks has been found to improve the quality of the predictors used $[50,51]$. Despite its popularity, its drawbacks motivates the development of other oversampling methods [52].

Geometric SMOTE (G-SMOTE) [52] introduces a modification of the SMOTE algorithm in the data generation mechanism to produce artificial instances with higher variability. Instead of generating artificial data as a linear combination of the parent instances, it is done within a deformed, truncated hyper-spheroid. G-SMOTE generates an artificial instance $\vec{z}$ within a hyper-spheroid, formed by selecting a minority instance $\vec{x}$ and one of its nearest neighbors $\vec{y}$, as shown in Figure 2. The truncation and deformation parameters define the shape of the spheroid's geometry. The method also modifies the selection strategy for the $k$-nearest neighbors, accepting the generation of artificial instances using instances from different classes, as shown in Figure 2. The modification of both selection and generation mechanisms addresses the main drawbacks found in SMOTE, the generation of both noisy data (i.e., the generation of minority class instances within majority class regions) and near-duplicate minority class instances [52]. G-SMOTE has shown superior performance when compared with other oversampling methods for LULC classification tasks, regardless of the classifier used [53].

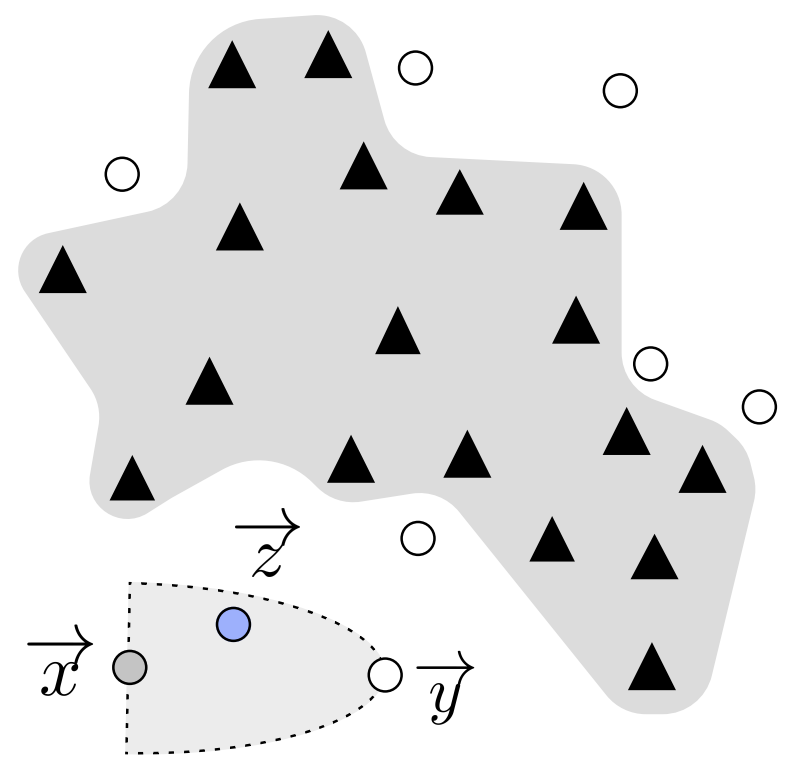

Figure 2. Example of G-SMOTE's generation process. G-SMOTE randomly selects instance $\vec{x}$ and one of its nearest neighbors $\vec{y}$ to produce instance $\vec{z}$.

\section{Proposed Method}

Within the literature identified, most of the work developed in the AL domain revolves around improving the quality of classification algorithms and/or selection criteria. Although these methods allow earlier convergence of the AL iterative process, the impact of these methods are only observed between iterations. Consequently, none of these contributions focus on the definition of decision borders within iterations. The method proposed in this paper modifies the AL framework by introducing an artificial data generation step within AL's iterative process. We define this component as the generator, and it is intended to be integrated into the AL framework, as shown in Figure 3.

This modification, by using a new source of data to augment the training set, leverages the data annotation work conducted by the human operator. The artificial data generated between iterations reduce the amount of labeled data required to reach optimal performance and lower the amount of human labor required to train a classifier to its optimal 
performance. This process lowers the annotation and overall training costs by translating some of the annotation cost into computational cost.
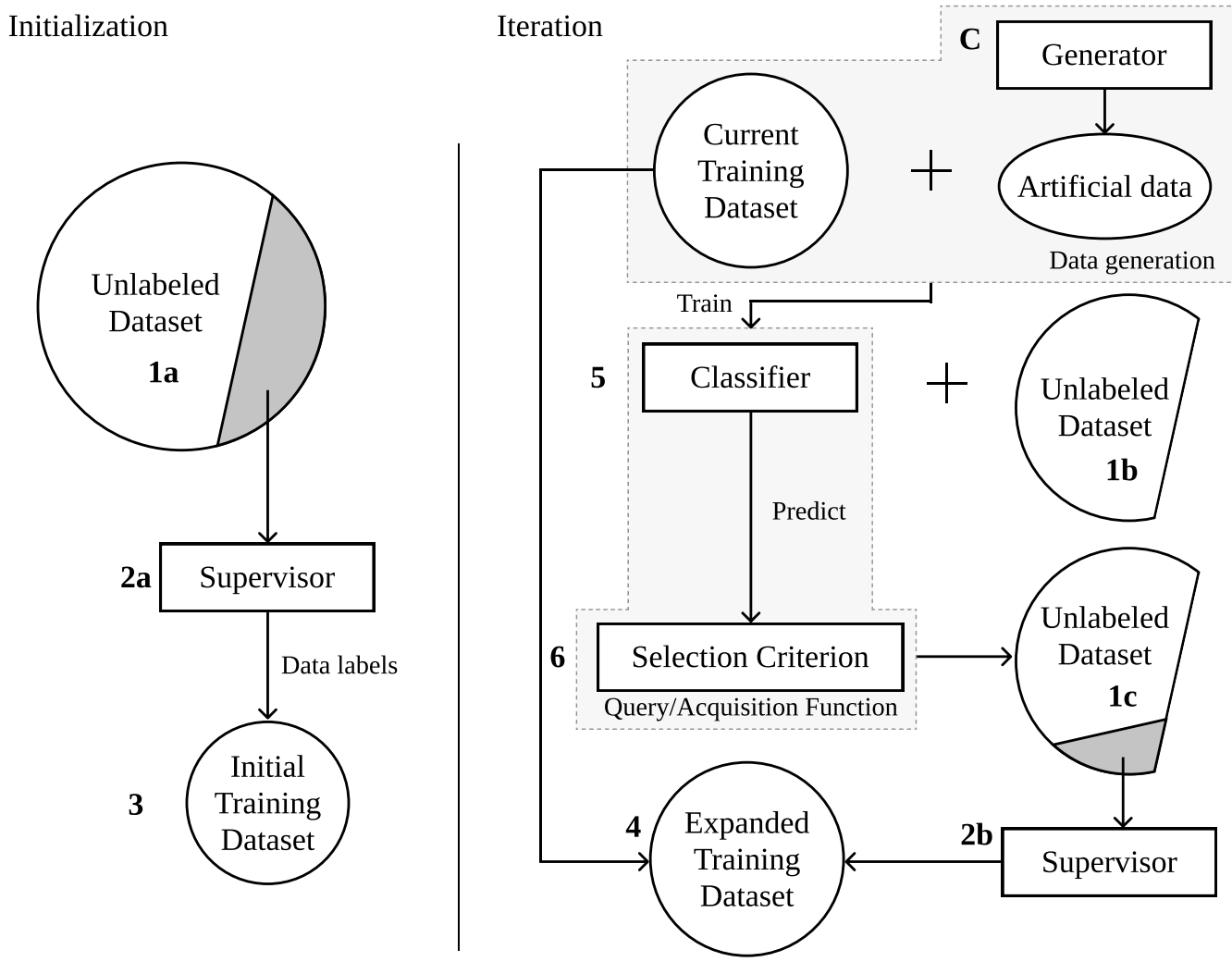

Figure 3. Proposed AL framework. This paper's contribution comprises a change in the AL framework through the introduction of a data generation mechanism, represented as the generator (marked with $C$ ), which is used to add artificial instances to the training dataset.

This method leverages the capability of artificial data to introduce more data variability into the augmented dataset and facilitate the chooser's training phase with a more consistent definition of the decision boundaries at each iteration. Therefore, any algorithm capable of producing artificial data, be it agnostic or specific to the domain, can be employed. The artificial data are only used to train the classifiers involved in the process and are discarded once the training phase is completed. The remaining steps in the AL framework remain unchanged. This method addresses the limitations found in the previous sections:

1. The convergence of classification performance should be anticipated with the clearer definition of the decision boundaries across iterations.

2. Annotation cost is expected to be reduced as the need for labeled instances reduces along with the early convergence of the classification performance.

3. The class imbalance bias observed in typical classification tasks, as well as in AL, is mitigated by balancing the class frequencies at each iteration.

Although the performance of this method is shown within a LULC classification context, the proposed framework is independent of the domain. The high dimensionality of remotely sensed imagery makes its classification particularly challenging when the available labeled data are scarce and/or come at a high cost, being subjected to the curse of dimensionality. Consequently, it is a relevant and appropriate domain to test this method. 


\section{Methodology}

In this section, we describe the datasets, evaluation metrics, oversampler, classifiers, software used and the procedure developed. We demonstrate the proposed method's efficiency over seven datasets, sampled from publicly available, well-known remote sensing hyperspectral scenes frequently found in the remote sensing literature. The datasets and sampling strategy are described in Section 5.1. On each of these datasets, we apply three different classifiers over the entire training set to estimate the optimal classification performance, the original AL framework as the baseline reference and the proposed method using G-SMOTE as a generator, described in Section 5.2. The metrics used to estimate the performance of these algorithms are described in Section 5.3. Finally, the experimental procedure is described in Section 5.4.

Our methodology focuses on two objectives: (1) comparison of optimal classification performance among active learners and traditional supervised learning; and (2) comparison of classification convergence efficiency among AL frameworks.

\subsection{Datasets}

The datasets used were extracted from publicly available repositories containing hyperspectral images and ground truth data. Additionally, all datasets were collected using the same sampling procedure. The description of the hyperspectral scenes used in this study is provided in Table 1. These scenes were chosen because of their popularity in the research community and their high baseline classification scores. Consequently, demonstrating an outperforming method in this context is particularly challenging and valuable.

Table 1. Description of the hyperspectral scenes used in this experiment. The column "Res. (m)" refers to the resolution of the sensors (in meters) that captured each of the scenes.

\begin{tabular}{ccccccc}
\hline Dataset & Sensor & Location & Dimension & Bands & Res. (m) & Classes \\
\hline Botswana & Hyperion & Okavango Delta & $1476 \times 256$ & 145 & 30 & 14 \\
Salinas A & AVIRIS & California USA & $86 \times 83$ & 224 & 3.7 & 6 \\
Kennedy Space Center & AVIRIS & Florida USA & $512 \times 614$ & 176 & 18 & 16 \\
Indian Pines & AVIRIS & NW Indiana USA & $145 \times 145$ & 220 & 20 & 16 \\
Salinas & AVIRIS & California USA & $512 \times 217$ & 224 & 3.7 & 16 \\
Pavia University & ROSIS & Pavia Italy & $610 \times 610$ & 103 & 1.3 & 9 \\
Pavia Centre & ROSIS & Pavia Italy & $1096 \times 1096$ & 102 & 1.3 & 9 \\
\hline
\end{tabular}

The Indian Pines scene [54] is composed of agriculture fields in approximately two thirds of its coverage and low density built up areas and natural perennial vegetation in the remainder of its area (see Figure 4a). The Pavia Centre and University scenes are hyperspectral, high-resolution images containing ground truth data composed of urbanrelated coverage (see Figure $4 \mathrm{~b}, \mathrm{c}$ ). The Salinas and Salinas A scenes contain at-sensor radiance data. As a subset of Salinas, the Salinas A scene contains contains the vegetable fields present in Salinas, and the latter is also composed of bare soils and vineyard fields (see Figure $4 \mathrm{~d}, \mathrm{e}$ ). The Botswana scene contains ground truth data composed of seasonal swamps, occasional swamps, and drier woodlands located in the distal portion of the delta (see Figure 4f). The Kennedy Space Center scene contains a ground truth composed of both vegetation and urban-related coverage (see Figure $4 \mathrm{~g}$ ). 

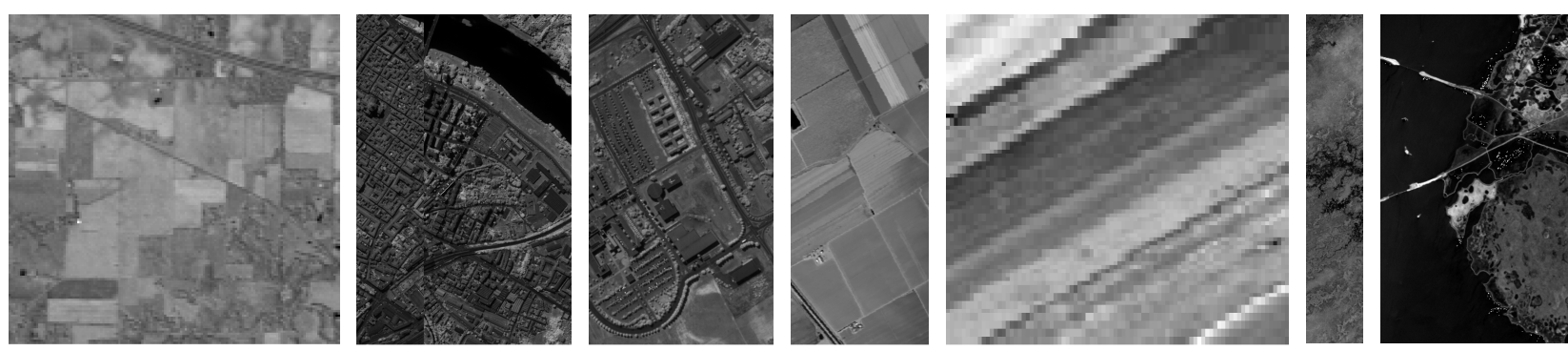

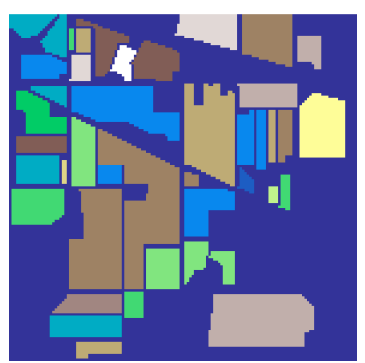

(a)

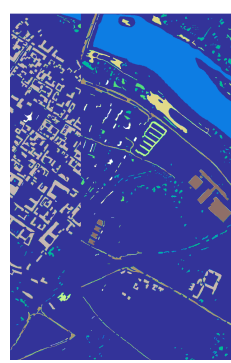

(b)

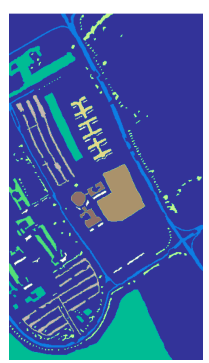

(c)

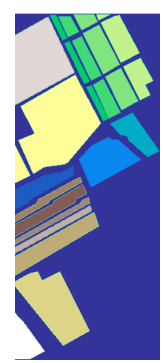

(d)

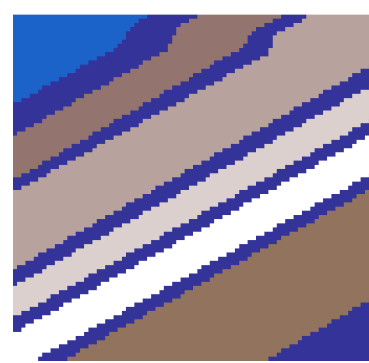

(e)

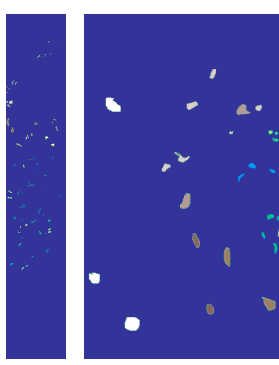

(f)

(g)

Figure 4. Gray scale visualization of a band (top row) and ground truth (bottom row) of each scene used in this study: (a) Indian Pines; (b) Pavia Centre; (c) Pavia University; (d) Salinas; (e) Salinas A; (f) Botswana; and (g) Kennedy Space Center.

The sampling strategy is similar for all datasets. The pixels without a ground truth label are first discarded. All the classes with cardinality lower than 150 are also discarded. This is done to maintain feasible Imbalance Ratios (IR) across datasets (where $\left.I R=\frac{\operatorname{count}\left(C_{m a j}\right)}{\operatorname{count}\left(C_{\min }\right)}\right)$. Finally, a stratified sample of 1500 instances are selected for the experiment. The resulting datasets are described in Table 2. The motivation for this strategy is three fold: (1) reduce the datasets to a manageable size and allow the experimental procedure to be completed within a feasible time frame; (2) ensure the relative class frequencies in the scenes are preserved; and (3) ensure equivalent analyses across datasets and AL frameworks. In this context, a fixed number of instances per dataset is especially important to standardize the AL-related performance metrics.

Table 2. Description of the datasets collected from each corresponding scene. The sampling strategy is similar to all scenes.

\begin{tabular}{|c|c|c|c|c|c|c|}
\hline Dataset & Features & Instances & Min. Instances & Maj. Instances & IR & Classes \\
\hline Botswana & 145 & 1500 & 89 & 154 & 1.73 & 12 \\
\hline Salinas A & 224 & 1500 & 109 & 428 & 3.93 & 6 \\
\hline Kennedy Space Center & 176 & 1500 & 47 & 272 & 5.79 & 12 \\
\hline Indian Pines & 220 & 1500 & 31 & 366 & 11.81 & 12 \\
\hline Salinas & 224 & 1500 & 25 & 312 & 12.48 & 16 \\
\hline Pavia University & 103 & 1500 & 33 & 654 & 19.82 & 9 \\
\hline Pavia Centre & 102 & 1500 & 27 & 668 & 24.74 & 9 \\
\hline
\end{tabular}

\subsection{Machine Learning Algorithms}

We use two different types of ML algorithms: a data generation algorithm to form the generator and a classification algorithms to calculate the classification uncertainties in the unlabeled dataset and predict the class labels in the validation and test sets.

Although any method capable of generating artificial data can be used as a generator, the one used in this experiment is an oversampler, originally developed to deal with imbalanced learning problems. Specifically, we chose G-SMOTE, a state-of-the-art oversampler. 
Three classification algorithms are used. We use different types of classifiers to test the framework's performance under varying situations: neighbors-based, linear and ensemble models. The neighbors-based classifier chosen is $K$-nearest neighbors (KNN) [55], a logistic regression (LR) [56] is used as the linear model, and a random forest classifier (RFC) [57] is used as the ensemble model.

The acquisition function is completed by testing three different selection criteria. Random selection is used as a baseline selection criterion, whereas entropy and breaking ties are used due to their popularity and independence of the classifier used.

\subsection{Evaluation Metrics}

Since the datasets used in this experiment have an imbalanced distribution of class frequencies, metrics such as the Overall Accuracy (OA) and Kappa coefficient are insufficient to accurately depict classification performance [58,59]. Instead, metrics such as Producer's Accuracy (or Recall) and User's Accuracy (or Precision) can be used. Since they consist of ratios based on True/False Positives (TP and FP) and Negatives (TN and FN), they provide per class information regarding the classifier's classification performance. However, in this experiment, the meaning and number of classes available in each dataset vary, making these metrics difficult to synthesize.

The performance metric Geometric mean (G-mean) and F-score are less sensitive to the data imbalance bias [60,61]. Therefore, we employ both of these scorers. G-mean consists of the geometric mean of Specificity $=\frac{T N}{T N+F P}$ and Sensitivity $=\frac{T P}{T P+F N}$ (also known as Recall) [61]. Both metrics are calculated in a multi-class context considering a one-versus-all approach. For multi-class problems, the G-mean scorer is calculated as its average of per class values:

$$
\text { G-mean }=\sqrt{\overline{\text { Sensitivity }}_{i} \times \overline{\text { Specificity }}_{i}}
$$

The F-score performance metric is the harmonic mean of Precision and Recall. The two metrics are also calculated considering a one-versus-all approach. The F-score for the multi-class case can be calculated using its average per class values [62]:

$$
\text { F-score }=2 \frac{\overline{\text { Precision }} \times \overline{\text { Recall }}}{\overline{\text { Precision }}+\overline{\text { Recall }}}
$$

The comparison of classification convergence across AL frameworks and selection criteria is done using two AL-specific performance metrics. Particularly, we follow the recommendations found in [22]. Each AL configuration is evaluated using the Area Under the Learning Curve (AULC) performance metric. It is the sum of the classification performance values of all iterations. To facilitate the analysis of the results, we fix the range of this metric within $[0,1]$ by dividing it by the total number of iterations (i.e., the maximum performance area).

The Data Utilization Rate (DUR) [63] metric consists of the ratio between the number of instances required to reach a given G-mean score threshold by an AL strategy and an equivalent baseline strategy. For easier interpretability, we simplify this metric by using the percentage of training data used by an AL strategy to reach the performance threshold, instead of presenting these values as a ratio of the baseline strategy. The DUR metric is measured at nine different performance levels, between 0.6 and 0.95 G-mean scores at a 0.05 step.

\subsection{Experimental Procedure}

A common practice in methodological evaluations is the implementation of an offline experiment [64]. It consists of using an existing set of labeled data as a proxy for the population of unlabeled instances. Because the dataset is already fully labeled, the supervisor's typical annotation process involved in each iteration is done at zero cost. Each AL and classifier configuration is tested using a stratified five-fold cross validation testing scheme. For each round, the larger partition is split in a stratified fashion to form a 
training and validation set (containing $20 \%$ of the original partition). The validation set is used to evaluate the convergence efficiency of active learners; the chooser's classification performance metrics and amount of data points used at each iteration are used to compute the AULC and DUR. Additionally, within the AL iterative process, the classifier with optimal performance on the validation set is evaluated using the test set. In order to further reduce possible initialization biases, this procedure is repeated three times with different initialization seeds and the results of all runs are averaged (i.e., each configuration is trained and evaluated 15 times). Finally, the maximum performance lines are calculated using the same approach. In those cases, the validation set is not used. The experimental procedure is depicted in Figure 5.

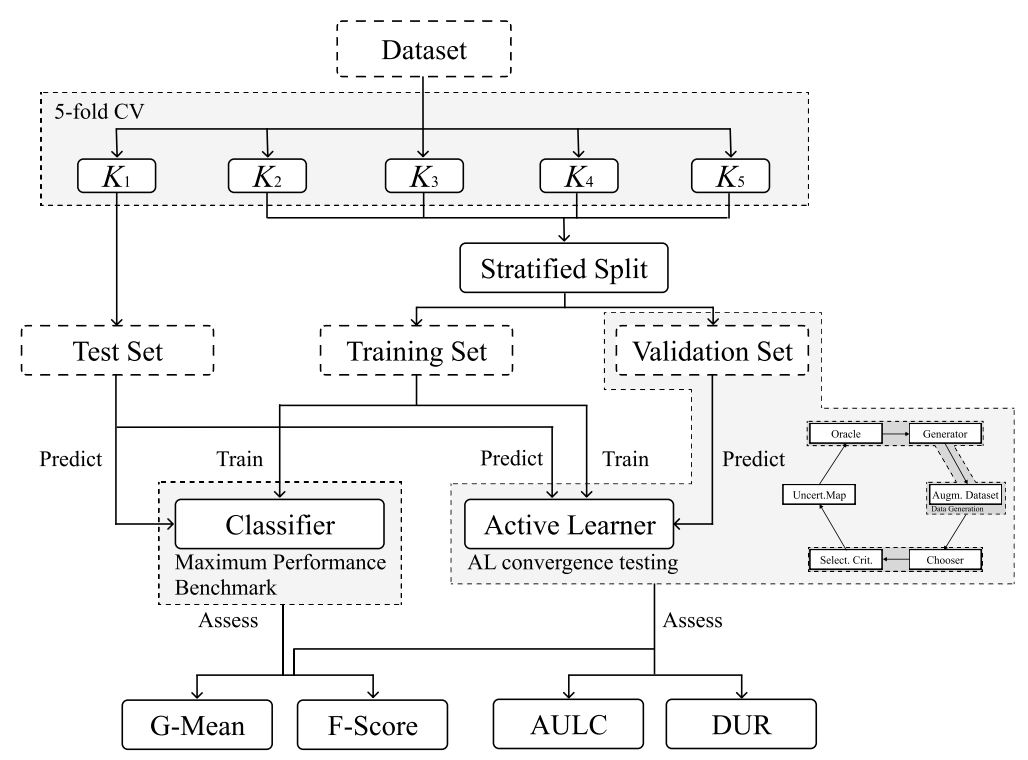

Figure 5. Experimental procedure. The datasets extracted from hyperspectral scenes are split into five folds. One of those (e.g., $K_{1}$ ) is used to test the optimal performance of AL algorithms and the classification without AL. The training set is used to iterate AL algorithms and train classifiers. The validation set is used to test the convergence of AL algorithms. The results are averaged over the five folds across each of the three different initializations of this procedure.

To make the AL-specific metrics comparable among active learners, the configurations of the different frameworks must be similar. For each dataset, the number of instances is constant to facilitate the analysis of the same metrics.

In most practical $\mathrm{AL}$ applications, it is assumed that the number of instances in the initial training sample is too small to perform hyperparameter tuning. Consequently, in order to ensure realistic results, our experimental procedure does not include hyperparameter optimization. The predefined hyperparameters are shown in Table 3 . They are set up based on general recommendations and default settings for the classifiers and generators used.

The AL iterative process is set up with a randomly selected initial training sample with 15 initial samples. At each iteration, 15 additional samples are added to the training set. This process is stopped after 49 iterations, once $50 \%$ of the entire dataset (i.e., $78 \%$ of the training set) is added to the augmented dataset. 
Table 3. Hyper-parameter definition for the classifiers and generator used in the experiment.

\begin{tabular}{lll}
\hline Classifier & Hyperparameters & Values \\
\hline LR & maximum iterations & 10,000 \\
& solver & sag \\
KNN & \# nenalty & None \\
& weights & 5 \\
& metric & uniform \\
RF & maximum tree depth & euclidean \\
& \# estimators & None \\
& criterion & 100 \\
Generator & & gini \\
\hline G-SMOTE & \# neighbors & \\
& deformation factor & 5 \\
& truncation factor & 0.5 \\
\hline
\end{tabular}

\subsection{Software Implementation}

The experiment was implemented using the Python programming language, along with the Python libraries Scikit-Learn (https://scikit-learn.org/stable/ (accessed on 2 July 2021)) [65], Imbalanced-Learn (https:/ / imbalanced-learn.org/en/stable/ (accessed on 2 July 2021)) [66], Geometric-SMOTE (https://geometric-smote.readthedocs.io/en/ latest/?badge=latest (accessed on 2 July 2021)), Cluster-Over-Sampling (https: / / clusterover-sampling.readthedocs.io/en/latest/?badge=latest (accessed on 2 July 2021)), and Research-Learn (https:/ / research-learn.readthedocs.io/en/latest/ ?badge=latest (accessed on 2 July 2021)). All functions, algorithms, experiments, and results are provided in the GitHub repository of the project (https://github.com/joaopfonseca/research/ (accessed on 2 July 2021)).

\section{Results and Discussion}

The evaluation of the different AL frameworks in a multiple dataset context should not rely uniquely on the mean of the performance metrics across datasets. Demšar [67] recommended the use of mean ranking scores, since the performance levels of the different frameworks varies according to the data it is being used on. Consequently, evaluating these performance metrics solely based on their mean values might lead to inaccurate analyses. Accordingly, the results of this experiment are analyzed using both the mean ranking and absolute scores for each model. The rank values are assigned based on the mean scores resulting from three different initializations of five-fold cross validation for each classifier and active learner. The goal of this analysis is to understand whether the proposed framework (AL with the integration of an artificial data generator) is capable of using fewer data from the original dataset while simultaneously achieving better classification results than the standard AL framework, i.e., guarantee a faster classification convergence.

\subsection{Results}

Table 4 shows the average rankings and standard deviations across datasets of the AULC scores for each active learner.

The mean AULC absolute scores are provided in Table 5. These values are computed as the mean of the sum of the scores of a specific performance metric over all iterations (for an AL configuration). In other words, these values correspond to the average AULC over 7 datasets $\times 5$ folds $\times 3$ initializations.

The average DURs are shown in Table 6. They were calculated for various G-mean scores thresholds, varying at a step of $5 \%$ between $60 \%$ and $95 \%$. Each row shows the percentage of training data required by the different AL configurations to reach that specific G-mean score. 
Table 4. Mean rankings of the AULC metric over the different datasets (7), folds (5), and runs (3) used in the experiment. This means that the use of G-SMOTE almost always improves the results of the original framework.

\begin{tabular}{cccc}
\hline Classifier & Evaluation Metric & Standard & Proposed \\
\hline KNN & F-score & $2.00 \pm 0.0$ & $1.00 \pm 0.0$ \\
KNN & G-mean & $2.00 \pm 0.0$ & $1.00 \pm 0.0$ \\
LR & F-score & $1.71 \pm 0.45$ & $1.29 \pm 0.45$ \\
LR & G-mean & $2.00 \pm 0.0$ & $1.00 \pm 0.0$ \\
RF & F-score & $1.86 \pm 0.35$ & $1.14 \pm 0.35$ \\
RF & G-mean & $2.00 \pm 0.0$ & $1.00 \pm 0.0$ \\
\hline
\end{tabular}

Table 5. Average AULC of each AL configuration tested. Each AULC score is calculated using the G-mean scores of each iteration in the validation set. By the end of the iterative process, each AL configuration used a total of 750 of the 960 instances that compose the training set.

\begin{tabular}{cccc}
\hline Classifier & Evaluation Metric & Standard & Proposed \\
\hline KNN & F-score & $0.762 \pm 0.131$ & $0.794 \pm 0.123$ \\
KNN & G-mean & $0.864 \pm 0.079$ & $0.886 \pm 0.073$ \\
LR & F-score & $0.839 \pm 0.119$ & $0.843 \pm 0.116$ \\
LR & G-mean & $0.907 \pm 0.074$ & $0.911 \pm 0.071$ \\
RF & F-score & $0.810 \pm 0.109$ & $0.819 \pm 0.1$ \\
RF & G-mean & $0.890 \pm 0.068$ & $0.901 \pm 0.059$ \\
\hline
\end{tabular}

Table 6. Mean data utilization of AL algorithms, as a percentage of the training set.

\begin{tabular}{cccc}
\hline G-Mean Score & Classifier & Standard & Proposed \\
\hline 0.60 & KNN & $4.0 \%$ & $2.1 \%$ \\
0.60 & LR & $2.2 \%$ & $2.1 \%$ \\
0.60 & RF & $2.2 \%$ & $2.1 \%$ \\
0.65 & KNN & $5.6 \%$ & $2.8 \%$ \\
0.65 & LR & $3.0 \%$ & $2.7 \%$ \\
0.65 & RF & $3.1 \%$ & $2.6 \%$ \\
0.70 & KNN & $7.9 \%$ & $4.1 \%$ \\
0.70 & LR & $4.2 \%$ & $4.1 \%$ \\
0.70 & RF & $4.5 \%$ & $3.6 \%$ \\
0.75 & KNN & $13.5 \%$ & $7.1 \%$ \\
0.75 & LR & $7.2 \%$ & $6.6 \%$ \\
0.75 & RF & $6.6 \%$ & $5.4 \%$ \\
0.80 & KNN & $24.4 \%$ & $16.9 \%$ \\
0.80 & LR & $13.1 \%$ & $11.7 \%$ \\
0.80 & RF & $11.6 \%$ & $9.2 \%$ \\
0.85 & KNN & $29.8 \%$ & $23.6 \%$ \\
0.85 & LR & $19.8 \%$ & $18.8 \%$ \\
0.85 & RF & $23.1 \%$ & $17.3 \%$ \\
0.90 & KNN & $41.0 \%$ & $36.1 \%$ \\
0.90 & LR & $28.1 \%$ & $24.8 \%$ \\
0.90 & RF & $37.1 \%$ & $30.3 \%$ \\
0.95 & KNN & $71.3 \%$ & $69.1 \%$ \\
0.95 & LR & $45.8 \%$ & $40.2 \%$ \\
0.95 & RF & $64.6 \%$ & $62.2 \%$ \\
\hline
\end{tabular}

The DUR of the proposed method relative to the baseline method is shown in Figure 6. A DUR below 1 means that the proposed framework requires fewer data to reach the same performance threshold (as a percentage, relative to the amount of data required by the 
baseline framework). For instance, in the upper left graphic, we can see that the proposed framework achieves $90 \%$ classification using F-score while using $91 \%$ of the amount of data used by the traditional AL framework, in other words $9 \%$ fewer data.

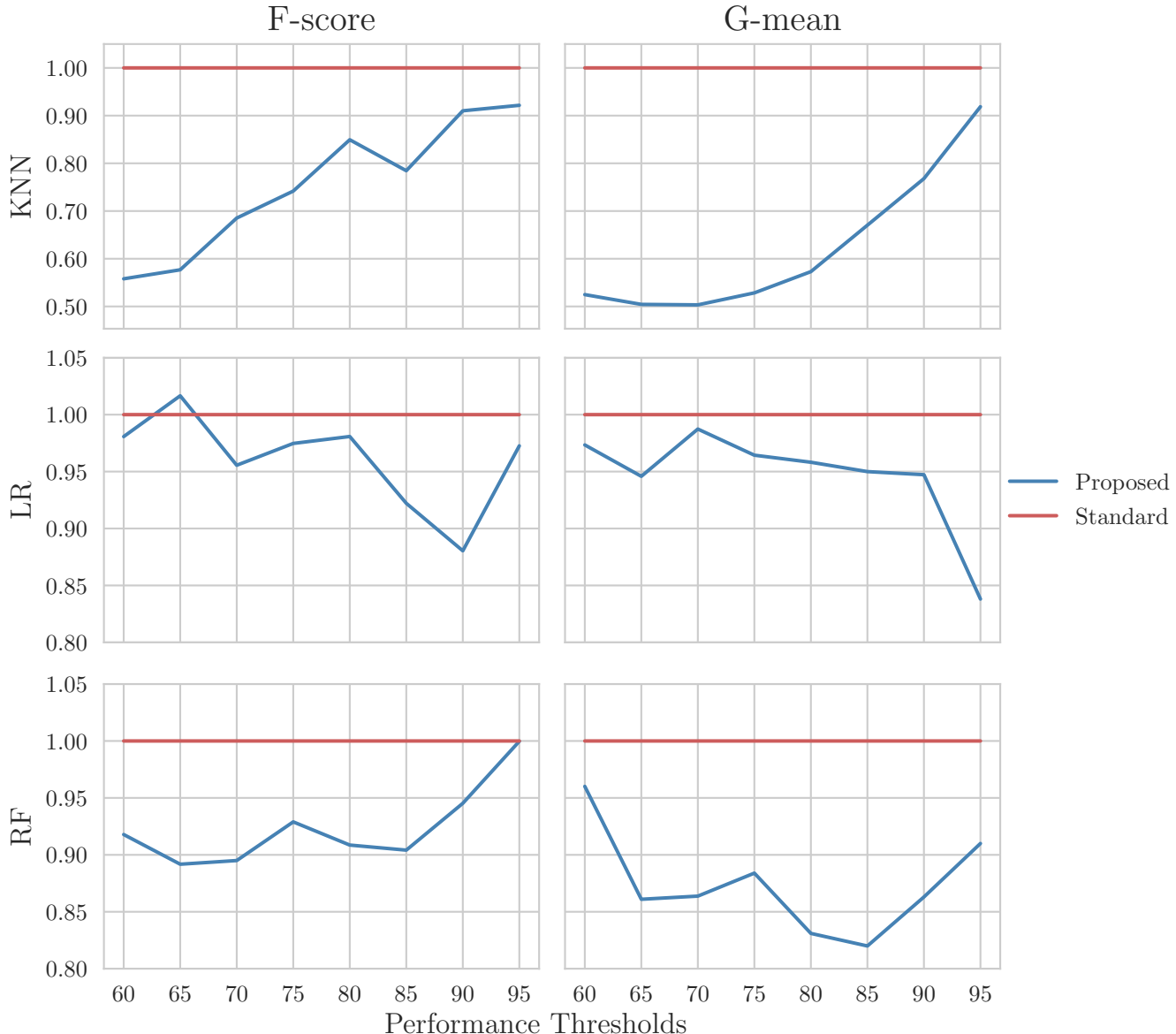

Figure 6. Mean data utilization rates. The y-axis shows the percentage of data (relative to the baseline AL framework) required to reach the different performance thresholds.

The averaged optimal classification scores are shown in Table 7. The maximum performance (MP) classification scores are shown as a benchmark and represent the performance of the corresponding classifier using the entire training set.

Table 7. Optimal classification scores. The Maximum Performance (MP) classification scores are calculated using classifiers trained using the entire training set.

\begin{tabular}{ccccc}
\hline Classifier & Evaluation Metric & MP & Standard & Proposed \\
\hline KNN & F-score & $0.838 \pm 0.106$ & $0.835 \pm 0.115$ & $0.843 \pm 0.105$ \\
KNN & G-mean & $0.907 \pm 0.063$ & $0.904 \pm 0.069$ & $0.912 \pm 0.061$ \\
LR & F-score & $0.890 \pm 0.084$ & $0.883 \pm 0.096$ & $0.887 \pm 0.097$ \\
LR & G-mean & $0.935 \pm 0.052$ & $0.931 \pm 0.059$ & $0.938 \pm 0.055$ \\
RF & F-score & $0.859 \pm 0.083$ & $0.866 \pm 0.081$ & $0.869 \pm 0.08$ \\
RF & G-mean & $0.918 \pm 0.051$ & $0.921 \pm 0.051$ & $0.930 \pm 0.043$ \\
\hline
\end{tabular}

\subsection{Statistical Analysis}

The methods used to test the experiment's results must be appropriate for a multidataset context. Therefore, the statistical analysis is performed using the Wilcoxon signedrank test [68] as a post hoc analysis. The variable used for this test is the data utilization rate 
based on the G-mean performance metric, considering the various performance thresholds in Table 6.

The Wilcoxon signed-rank test results are shown in Table 8 . We test as null hypothesis that the performance of the proposed framework is the same as the original AL framework. The null hypothesis is rejected in all datasets.

Table 8. Adjusted p-values using the Wilcoxon signed-rank method. Bold values are statistically significant at a level of $\alpha=0.05$. The null hypothesis is that the performance of the proposed framework is similar to that of the original framework.

\begin{tabular}{ccc}
\hline Dataset & $p$-Value & Significance \\
\hline Botswana & $3.8 \times 10^{-3}$ & TRUE \\
Indian Pines & $2.3 \times 10^{-4}$ & TRUE \\
Kennedy Space Center & $1.3 \times 10^{-4}$ & TRUE \\
Pavia Centre & $4.3 \times 10^{-3}$ & TRUE \\
Pavia University & $4.6 \times 10^{-5}$ & TRUE \\
Salinas & $4.6 \times 10^{-5}$ & TRUE \\
Salinas A & $3.0 \times 10^{-3}$ & TRUE \\
\hline
\end{tabular}

\subsection{Discussion}

This paper expands the AL framework by adding an artificial data generator into its iterative process. This modification is done to accelerate the classification convergence of the standard AL procedure, which is reflected in the reduction of the amount of data necessary to reach better classification results.

The convergence efficiency of the proposed method is always higher than the baseline AL framework, with the exception of one comparison, as shown in Table 4 and Figure 6. This means the proposed AL framework using data generation was able to outperform the baseline AL in nearly all scenarios.

The mean AULC scores in Table 5 show a significant improvement in the performance of AL when a generator is used. The mean performance of the proposed framework is always better than the baseline framework. This improvement is explained by:

1. There is earlier convergence of AL, i.e., it requires fewer data to achieve comparable performance levels. This effect is shown in Table 6, where we found that the proposed framework always uses fewer data for similar performance levels, regardless of the classifier used.

2. There is higher optimal classification performance, i.e., it reaches higher performance levels overall. This effect is shown in Table 7, where we found that using a generator in AL led to a better classification performance and the capability of outperforming the MP threshold.

Our results show statistical significance in every dataset. The proposed framework had a superior performance with statistical significance on each dataset at a level of $\alpha=0.05$. This indicates that, regardless of the context under which an AL algorithm is used, the proposed framework reduces the amount of data necessary in the AL's iterative process.

This paper introduces the concept of applying a data generation algorithm in the AL framework. This was done with the implementation of a recent state-of-the-art generalization of a popular data generation algorithm. However, since this algorithm is based on heuristics, future work should focus on improving these results through the design of new data generation mechanisms, at the cost of additional computational power. In addition, we also noticed significant standard errors in our experimental results (see Section 6.1). This indicates that AL procedures seem to be particularly sensitive to the initialization method, which is still a limitation of AL, regardless of the framework and configurations used. This is consistent with the findings in [22], which future work should attempt to address. Although using a generator marginally reduced this standard error, it is not sufficient to address this specific limitation. 


\section{Conclusions}

The aim of this experiment was to test the effectiveness of a new AL framework that introduces artificial data generation in its iterative process. The experiment was designed to test the proposed method under particularly challenging conditions, where the maximum performance line is naturally high in most datasets. The element that constitutes the generator component was set up in a plug-and-play scheme, without significant tuning of the G-SMOTE oversampler. Using a generator in AL improved the original AL framework in all scenarios. These results could be further improved through the modification and more intense tuning of the data generation strategy. In our experiment, artificial data were generated only to match each non-majority class frequency with the majority class frequency, strictly balancing the class distribution. Generating a larger amount of data for all classes can further improve these results.

The high performance scores for the baseline AL framework made the achievement of significant improvements over the traditional AL framework under these conditions particularly meaningful. The advantage of the proposed AL framework is shown in Table 6 . In most of the presented scenarios, there is a substantial reduction of data necessary to reach a given performance threshold.

The results from this experiment show that using a data generator in the AL framework will improve the convergence of the method. This framework successfully anticipates the predictor's optimal performance, as shown in Tables 4-6. Therefore, in a real application, the annotation cost would have been reduced since fewer iterations and labeled instances are necessary to reach near optimal classification performance.

Author Contributions: Conceptualization, F.B.; Methodology, J.F. and G.D.; Software, J.F. and G.D.; Validation, F.B. and G.D.; Formal Analysis, J.F.; Writing-Original Draft Preparation, J.F.; WritingReview and Editing, F.B., G.D. and J.F.; Supervision, F.B.; and Funding Acquisition, F.B. All authors have read and agreed to the published version of the manuscript.

Funding: This research was funded by "Fundação para a Ciência e a Tecnologia" (Portugal) [grant numbers PCIF/SSI/0102/2017_foRESTER, DSAIPA/AI/0100/2018_IPSTERS].

Institutional Review Board Statement: Not applicable.

Informed Consent Statement: Not applicable.

Data Availability Statement: The data reported in this study are publicly available. They can be retrieved and preprocessed using the Python source code provided at (https://github.com/ joaopfonseca/research (accessed on 2 July 2021)). Alternatively the original data are available at http:/ / www.ehu.eus / ccwintco/index.php?title=Hyperspectral_Remote_Sensing_Scenes (accessed on 2 July 2021).

Acknowledgments: The authors would like to thank Professor Victor Lobo (NOVA IMS, Universidade Nova de Lisboa, and CINAV, Escola Naval, CIDIUM) for reviewing this paper and providing important feedback throughout its development.

Conflicts of Interest: The authors declare no conflict of interest. The funders had no role in the design of the study; in the collection, analyses, or interpretation of data; in the writing of the manuscript, or in the decision to publish the results.

\section{References}

1. Nagai, S.; Nasahara, K.N.; Akitsu, T.K.; Saitoh, T.M.; Muraoka, H. Importance of the Collection of Abundant Ground-Truth Data for Accurate Detection of Spatial and Temporal Variability of Vegetation by Satellite Remote Sensing. In Biogeochemical Cycles: Ecological Drivers and Environmental Impact; American Geophysical Union (AGU): Washington, DC, USA, 2020; pp. 223-244. [CrossRef]

2. Huang, Y.; Chen, X.Z.; YU, T.; Huang, X.Z.; Gu, X.F. Agricultural remote sensing big data: Management and applications. J. Integr. Agric. 2018, 17, 1915-1931. [CrossRef]

3. Wang, X.; Xie, H. A review on applications of remote sensing and geographic information systems (GIS) in water resources and flood risk management. Water 2018, 10, 608. [CrossRef] 
4. Khatami, R.; Mountrakis, G.; Stehman, S.V. A meta-analysis of remote sensing research on supervised pixel-based land-cover image classification processes: General guidelines for practitioners and future research. Remote Sens. Environ. 2016, 177, 89-100. [CrossRef]

5. Gavade, A.B.; Rajpurohit, V.S. Systematic analysis of satellite image-based land cover classification techniques: Literature review and challenges. Int. J. Comput. Appl. 2019, 1-10. [CrossRef]

6. Wulder, M.A.; Coops, N.C.; Roy, D.P.; White, J.C.; Hermosilla, T. Land cover 2.0. Int. J. Remote Sens. 2018, 39, 4254-4284. [CrossRef]

7. Costa, H.; Benevides, P.; Marcelino, F.; Caetano, M. Introducing automatic satellite image processing into land cover mapping by photo-interpretation of airborne data. Int. Arch. Photogramm. Remote Sens. Spat. Inf. Sci. 2020, 42, 29-34. [CrossRef]

8. Vermote, E.F.; Skakun, S.; Becker-Reshef, I.; Saito, K. Remote Sensing of Coconut Trees in Tonga Using Very High Spatial Resolution WorldView-3 Data. Remote Sens. 2020, 12, 3113. [CrossRef]

9. Costantino, D.; Pepe, M.; Dardanelli, G.; Baiocchi, V. Using Optical Satellite and Aerial Imagery for Automatic Coastline Mapping. Geogr. Tech. 2020, 15, 171-190. [CrossRef]

10. Maxwell, A.E.; Warner, T.A.; Fang, F. Implementation of machine-learning classification in remote sensing: An applied review. Int. J. Remote Sens. 2018, 39, 2784-2817. [CrossRef]

11. Růžička, V.; D’Aronco, S.; Wegner, J.D.; Schindler, K. Deep Active Learning in Remote Sensing for data efficient Change Detection. arXiv 2020, arXiv:2008.11201.

12. Liu, S.J.; Luo, H.; Shi, Q. Active Ensemble Deep Learning for Polarimetric Synthetic Aperture Radar Image Classification. IEEE Geosci. Remote Sens. Lett. 2020, 1-5. [CrossRef]

13. Su, T.; Zhang, S.; Liu, T. Multi-spectral image classification based on an object-based active learning approach. Remote Sens. 2020, 12, 504. [CrossRef]

14. Pasolli, E.; Yang, H.L.; Crawford, M.M. Active-metric learning for classification of remotely sensed hyperspectral images. IEEE Trans. Geosci. Remote Sens. 2016, 54, 1925-1939. [CrossRef]

15. Pelletier, C.; Valero, S.; Inglada, J.; Champion, N.; Sicre, C.M.; Dedieu, G. Effect of training class label noise on classification performances for land cover mapping with satellite image time series. Remote Sens. 2017, 9, 173. [CrossRef]

16. Stromann, O.; Nascetti, A.; Yousif, O.; Ban, Y. Dimensionality Reduction and Feature Selection for Object-Based Land Cover Classification based on Sentinel-1 and Sentinel-2 Time Series Using Google Earth Engine. Remote Sens. 2020, 12, 76. [CrossRef]

17. Alonso-Sarria, F.; Valdivieso-Ros, C.; Gomariz-Castillo, F. Isolation forests to evaluate class separability and the representativeness of training and validation areas in land cover classification. Remote Sens. 2019, 11, 3000. [CrossRef]

18. Feng, W.; Huang, W.; Ye, H.; Zhao, L. Synthetic minority over-sampling technique based rotation forest for the classification of unbalanced hyperspectral data. In Proceedings of the International Geoscience and Remote Sensing Symposium (IGARSS), Valencia, Spain, 22-27 July 2018; Institute of Electrical and Electronics Engineers Inc.: New York, NY, USA: 2018; Volume 2018; pp. 2651-2654. [CrossRef]

19. Chawla, N.V.; Japkowicz, N.; Kotcz, A. Editorial: Special Issue on Learning from Imbalanced Data Sets. ACM SIGKDD Explor. Newsl. 2004, 6, 1-6. [CrossRef]

20. Fernández, A.; López, V.; Galar, M.; del Jesus, M.J.; Herrera, F. Analysing the classification of imbalanced data-sets with multiple classes: Binarization techniques and ad-hoc approaches. Knowl.-Based Syst. 2013, 42, 97-110. [CrossRef]

21. Kaur, H.; Pannu, H.S.; Malhi, A.K. A systematic review on imbalanced data challenges in machine learning: Applications and solutions. ACM Comput. Surv. 2019, 52,1-36. [CrossRef]

22. Kottke, D.; Calma, A.; Huseljic, D.; Krempl, G.; Sick, B. Challenges of reliable, realistic and comparable active learning evaluation. In Proceedings of the CEUR Workshop Proceedings, Skopje, Macedonia, 18 September 2017; Volume 1924; pp. 2-14.

23. Sverchkov, Y.; Craven, M. A review of active learning approaches to experimental design for uncovering biological networks. PLoS Comput. Biol. 2017, 13, e1005466. [CrossRef]

24. Li, J.; Huang, X.; Chang, X. A label-noise robust active learning sample collection method for multi-temporal urban land-cover classification and change analysis. ISPRS J. Photogramm. Remote Sens. 2020, 163, 1-17. [CrossRef]

25. Yoo, D.; Kweon, I.S. Learning Loss for Active Learning. In Proceedings of the IEEE/CVF Conference on Computer Vision and Pattern Recognition (CVPR), Long Beach, CA, USA, 15 June 2019.

26. Aghdam, H.H.; Gonzalez-Garcia, A.; Lopez, A.; Weijer, J. Active learning for deep detection neural networks. In Proceedings of the IEEE International Conference on Computer Vision, Seoul, Korea, 27 October 2019; pp. 3671-3679. [CrossRef]

27. Cawley, G. Baseline Methods for Active Learning. In Proceedings of the Active Learning and Experimental Design workshop In conjunction with AISTATS 2010, Sardinia, Italy, 16 May 2011; Volume 16, pp. 47-57.

28. Li, X.; Guo, Y. Active learning with multi-label SVM classification. In Proceedings of the Twenty-Third International Joint Conference on Artificial Intelligence, Beijing, China, 3 August 2013; pp. 1479-1485.

29. Tuia, D.; Pasolli, E.; Emery, W.J. Using active learning to adapt remote sensing image classifiers. Remote Sens. Environ. 2011, 115, 2232-2242. [CrossRef]

30. Cao, X.; Yao, J.; Xu, Z.; Meng, D. Hyperspectral Image Classification with Convolutional Neural Network and Active Learning. IEEE Trans. Geosci. Remote Sens. 2020, 58, 4604-4616. [CrossRef]

31. Wu, X.; Li, W.; Hong, D.; Tian, J.; Tao, R.; Du, Q. Vehicle detection of multi-source remote sensing data using active fine-tuning network. ISPRS J. Photogramm. Remote Sens. 2020, 167, 39-53. [CrossRef] 
32. Bi, H.; Xu, F.; Wei, Z.; Xue, Y.; Xu, Z. An Active Deep Learning Approach for Minimally Supervised PolSAR Image Classification. IEEE Trans. Geosci. Remote Sens. 2019, 57, 9378-9395. [CrossRef]

33. Hu, L.; Robinson, C.; Dilkina, B. Model Generalization in Deep Learning Applications for Land Cover Mapping. arXiv 2020, arXiv:2008.10351.

34. Roy, S.K.; Krishna, G.; Dubey, S.R.; Chaudhuri, B.B. HybridSN: Exploring 3-D-2-D CNN Feature Hierarchy for Hyperspectral Image Classification. IEEE Geosci. Remote Sens. Lett. 2019, 1-5. [CrossRef]

35. Shrivastava, V.K.; Pradhan, M.K. Hyperspectral Remote Sensing Image Classification Using Active Learning. In Studies in Computational Intelligence; Springer: Cham, Switzerland, 2021; Volume 907; pp. 133-152. [CrossRef]

36. Muslea, I.; Minton, S.; Knoblock, C.A. Active learning with multiple views. J. Artif. Intell. Res. 2006, 27, 203-233. [CrossRef]

37. Di, W.; Crawford, M.M. View generation for multiview maximum disagreement based active learning for hyperspectral image classification. IEEE Trans. Geosci. Remote Sens. 2012, 50, 1942-1954. [CrossRef]

38. Zhou, X.; Prasad, S.; Crawford, M. Wavelet domain multi-view active learning for hyperspectral image analysis. In Proceedings of the Workshop on Hyperspectral Image and Signal Processing, Evolution in Remote Sensing, IEEE Computer Society, Lausanne, Switzerland, 24 June 2014. [CrossRef]

39. Zhang, Z.; Pasolli, E.; Yang, H.L.; Crawford, M.M. Multimetric Active Learning for Classification of Remote Sensing Data. IEEE Geosci. Remote Sens. Lett. 2016, 13, 1007-1011. [CrossRef]

40. Tuia, D.; Ratle, F.; Pacifici, F.; Kanevski, M.F.; Emery, W.J. Active learning methods for remote sensing image classification. IEEE Trans. Geosci. Remote Sens. 2009, 47, 2218-2232. [CrossRef]

41. Copa, L.; Tuia, D.; Volpi, M.; Kanevski, M. Unbiased query-by-bagging active learning for VHR image classification. In Image and Signal Processing for Remote Sensing XVI; Bruzzone, L., Ed.; SPIE Remote Sensing, Toulouse, France, 20 September 2010; Volume 7830, p. 78300K. [CrossRef]

42. Demir, B.; Persello, C.; Bruzzone, L. Batch-mode active-learning methods for the interactive classification of remote sensing images. IEEE Trans. Geosci. Remote Sens. 2011, 49, 1014-1031. [CrossRef]

43. Li, J.; Bioucas-Dias, J.M.; Plaza, A. Hyperspectral image segmentation using a new bayesian approach with active learning. IEEE Trans. Geosci. Remote Sens. 2011, 49, 3947-3960. [CrossRef]

44. Liu, W.; Yang, J.; Li, P.; Han, Y.; Zhao, J.; Shi, H. A novel object-based supervised classification method with active learning and random forest for PolSAR imagery. Remote Sens. 2018, 10, 1092. [CrossRef]

45. Luo, T.; Kramer, K.; Goldgof, D.; Hall, L.O.; Samson, S.; Remsen, A.; Hopkins, T. Learning to recognize plankton. In Proceedings of the IEEE International Conference on Systems, Man and Cybernetics, Washington, DC, USA, 5-8 October 2003; Volume 1, pp. 888-893. [CrossRef]

46. Li, J.; Bioucas-Dias, J.M.; Plaza, A. Spectral-spatial classification of hyperspectral data using loopy belief propagation and active learning. IEEE Trans. Geosci. Remote Sens. 2013, 51, 844-856. [CrossRef]

47. Ertekin, S.; Huang, J.; Giles, C.L. Active learning for class imbalance problem. In Proceedings of the 30th Annual International ACM SIGIR Conference on Research and Development in Information Retrieval, SIGIR'07, Amsterdam, The Netherlands, 23 July 2007; pp. 823-824. [CrossRef]

48. DeVries, T.; Taylor, G.W. Dataset augmentation in feature space. In Proceedings of the 5th International Conference on Learning Representations, ICLR 2017-Workshop Track Proceedings, International Conference on Learning Representations, ICLR, Toulon, France, 24-26 April 2017.

49. Chawla, N.V.; Bowyer, K.W.; Hall, L.O.; Kegelmeyer, W.P. SMOTE: Synthetic minority over-sampling technique. J. Artif. Intell. Res. 2002, 16, 321-357. [CrossRef]

50. Jozdani, S.E.; Johnson, B.A.; Chen, D. Comparing Deep Neural Networks, Ensemble Classifiers, and Support Vector Machine Algorithms for Object-Based Urban Land Use/Land Cover Classification. Remote Sens. 2019, 11, 1713. [CrossRef]

51. Bogner, C.; Seo, B.; Rohner, D.; Reineking, B. Classification of rare land cover types: Distinguishing annual and perennial crops in an agricultural catchment in South Korea. PLoS ONE 2018, 13. [CrossRef] [PubMed]

52. Douzas, G.; Bacao, F. Geometric SMOTE a geometrically enhanced drop-in replacement for SMOTE. Inf. Sci. 2019, 501, 118-135. [CrossRef]

53. Douzas, G.; Bacao, F.; Fonseca, J.; Khudinyan, M. Imbalanced learning in land cover classification: Improving minority classes' prediction accuracy using the geometric SMOTE algorithm. Remote Sens. 2019, 11, 3040. [CrossRef]

54. Baumgardner, M.F.; Biehl, L.L.; Landgrebe, D.A. 220 Band AVIRIS Hyperspectral Image Data Set: 12 June 1992 Indian Pine Test Site 3. Purdue Univ. Res. Repos. 2015. [CrossRef]

55. Cover, T.; Hart, P. Nearest neighbor pattern classification. IEEE Trans. Inf. Theory 1967, 13, 21-27. [CrossRef]

56. Nelder, J.A.; Wedderburn, R.W. Generalized linear models. J. R. Stat. Soc. Ser. A 1972, 135, 370-384. [CrossRef]

57. Ho, T.K. Random Decision Forests. In Proceedings of the Third International Conference on Document Analysis and Recognition (Volume 1), IEEE Computer Society, Montreal, QC, Canada, 14 August 1995; p. 278.

58. Olofsson, P.; Foody, G.M.; Stehman, S.V.; Woodcock, C.E. Making better use of accuracy data in land change studies: Estimating accuracy and area and quantifying uncertainty using stratified estimation. Remote Sens. Environ. 2013, 129, 122-131. [CrossRef]

59. Pontius, R.G.; Millones, M. Death to Kappa: Birth of quantity disagreement and allocation disagreement for accuracy assessment. Int. J. Remote Sens. 2011, 32, 4407-4429. [CrossRef] 
60. Jeni, L.A.; Cohn, J.F.; De La Torre, F. Facing imbalanced data-Recommendations for the use of performance metrics. In Proceedings of the 2013 Humaine Association Conference on Affective Computing and Intelligent Interaction, ACII 2013, Geneva, Switzerland, 2-5 September 2013; pp. 245-251. [CrossRef]

61. Kubat, M.; Matwin, S. Addressing the curse of imbalanced training sets: One-sided selection. In Proceedings of the International Conference on Machine Learning, Nashville, TN, USA, 8 July 1997; Volume 97; pp. 179-186.

62. He, H.; Garcia, E.A. Learning from Imbalanced Data. IEEE Trans. Knowl. Data Eng. 2009, 21, 1263-1284. [CrossRef]

63. Reitmaier, T.; Sick, B. Let us know your decision: Pool-based active training of a generative classifier with the selection strategy 4DS. Inf. Sci. 2013, 230, 106-131. [CrossRef]

64. Kagy, J.F.; Kayadelen, T.; Ma, J.; Rostamizadeh, A.; Strnadova, J. The Practical Challenges of Active Learning: Lessons Learned from Live Experimentation. arXiv 2019, arXiv:1907.00038.

65. Pedregosa, F.; Varoquaux, G.; Gramfort, A.; Michel, V.; Thirion, B.; Grisel, O.; Blondel, M.; Prettenhofer, P.; Weiss, R.; Dubourg, V.; et al. Scikit-learn: Machine Learning in Python. J. Mach. Learn. Res. 2011, 12, 2825-2830.

66. Lemaître, G.; Nogueira, F.; Aridas, C.K. Imbalanced-learn: A Python Toolbox to Tackle the Curse of Imbalanced Datasets in Machine Learning. J. Mach. Learn. Res. 2017, 18, 1-5.

67. Demšar, J. Statistical comparisons of classifiers over multiple data sets. J. Mach. Learn. Res. 2006, 7, 1-30.

68. Wilcoxon, F. Individual Comparisons by Ranking Methods. Biom. Bull. 1945, 1, 80. [CrossRef] 